\title{
The Effect of Postpartum Fatigue on Exclusive Breastfeeding Rates at Discharge: A Comparison of Birth Methods
}

Karen F Phillips EdD, MSN, RN*, Lisa DePue DNP, MSN, RNC-MNN, Spencer Mullen MA (c), BS

College of Nursing, William Paterson University, Wayne, NJ 07470, United States.

\author{
Article Details \\ Article Type: Research Article \\ Received date: $11^{\text {st }}$ January, 2020 \\ Accepted date: $29^{\text {th }}$ January, 2020 \\ Published date: $31^{\text {st }}$ January, 2020
}

"Corresponding Author: Karen F Phillips, Associate Professor, School of Nursing, William Paterson University, Wayne, NJ 07470, United States. E-mail: Phillipsk116@wpunj.edu

Citation: Phillips, K. F., DePue, L., Mullen, S. (2020). The effect of postpartum fatigue on exclusive breastfeeding rates at discharge: A comparison of birth methods. J Comp Nurs Res Care 5(1):158. doi: https://doi.org/10.33790/jenrc1100158.

Copyright: $(\mathcal{C} 2020$, This is an open-access article distributed under the terms of the Creative Commons Attribution License 4.0, which permits unrestricted use, distribution, and reproduction in any medium, provided the original author and source are credited.

\begin{abstract}
Exclusive breastfeeding is recommended for the first six months of life due to the benefits it offers to both baby and mother. Although breastfeeding initiation rates are high, breastfeeding continuation rates at six and twelve months and exclusive breastfeeding rates remain lower than Healthy People recommendations. The purpose of this study was to determine if postpartum fatigue had any impact on the success of exclusive breastfeeding when comparing birth methods. Although postpartum fatigue has been reported as a common occurrence, less is known about its effect on exclusive breastfeeding rates. A quasi-experimental study with 157 participants was completed to evaluate the effect of postpartum fatigue on exclusive breastfeeding rates at discharge using the Visual Analog Scale to Evaluate Fatigue (VAS-F). Both mothers who delivered vaginally and by cesarean section took part in this study at no less than one day after delivery. One hundred percent of mothers in the study reported varying levels of fatigue postpartum, with $47 \%$ breastfeeding exclusively. An independent samples T-test compared fatigue levels in vaginal and cesarean births. Statistical significance was noted in that cesarean section mothers were more fatigued than those who gave birth vaginally $(\mathrm{p}=0.015)$. Findings from a chi squared test showed exclusive breastfeeding rates in women who gave birth vaginally higher than cesarean section $\left(\chi^{2}=12.47, \mathrm{p}<.01\right)$ suggesting exclusive breastfeeding rates were dependent on birth type. Further research needs to be completed to determine what education or strategies relative to postpartum fatigue need to be identified to diminish the effects of postpartum fatigue which could lead to improved exclusive breastfeeding rates.
\end{abstract}

Keywords: Fatigue; Postpartum Fatigue, Breastfeeding and Postpartum Fatigue, Rest Periods and Postpartum Fatigue, Breastfeeding, Postpartum Fatigue and Birth Methods

\section{Introduction}

The changes a woman goes through after delivery have both psychological and physical effects during the postpartum period. One area of concern for the new mother is postpartum fatigue. Fatigue has been defined as a constant state of exhaustion impacting mental and physical abilities which even rest does not diminish [1]. Postpartum fatigue is experienced by nearly $64 \%$ of new mothers [2]. Postpartum women have described postpartum fatigue as a state of being exhausted and overwhelmed which impacted both their physiological and psychological capabilities creating a negative effect on the maternal-infant dyad $[2,3]$. Postpartum fatigue was also perceived as a negative experience that made relationships and everyday aspects difficult [4]. Postpartum fatigue has been a major concern of postpartum mothers for nearly two decades $[5,6]$.

The causes of postpartum fatigue have been found to be multifactorial. Physical, psychological, and situational factors have been reported to be causative agents in postpartum fatigue [5]. This study reviewed physical factors such as birth type, postpartum pain, and breastfeeding. Fatigue has been associated with both vaginal and cesarean birth [7]. Differences in fatigue levels have been reported between women giving birth vaginally and by cesarean section. A study by Kilic et al. [1] indicated that half of women who gave birth both vaginally and by cesarean reported moderate fatigue levels using the VAS-F scale. Perineal pain was determined to be a factor in fatigue related to vaginal birth due to effect of pain on a mother's ability to rest postpartum [8]. Lai et al. [7] reported that women rooming in with their newborns experienced more postpartum fatigue than those who gave birth vaginally.

Breastfeeding difficulties have also been associated with postpartum fatigue. Phillips et al. [9] conducted a study of 160 postpartum women examining postpartum fatigue and breastfeeding rates at discharge. The more exhaustion the mothers re-counted, the more likely they were to report breastfeeding difficulties [9]. Ahluwalia et al. [10] conducted a study of 3,026 women that investigated the relationship between method of delivery and breastfeeding initiation and continuation. The results showed that there was no difference in breastfeeding initiation rates; however, breastfeeding continuation differed greatly on method of delivery [10].A study by Lai et al. [7] reviewed data collected from 120 women in two different hospitals. The study findings suggested that women who delivered by cesarean birth were more likely to report greater postpartum fatigue [7]. Higher levels of postpartum fatigue were related to increased difficulty with parenting behaviors particularly with infant care and feeding [7].

The parenting behaviors of a mother may be less than adequate if a mother is experiencing postpartum fatigue [11]. One area of parenting for an infant that can only be provided by the mother is breastfeeding, which has been identified as the ideal source of nutrition for most infants $[12,13]$. Exclusive breastfeeding is recommended for the first six months of life $[12,13]$, as breast milk offers multiple benefits to mothers and babies [12]. For children, a reduction of asthma, childhood obesity, ear infections, eczema, and sudden infant death syndrome (SIDS) are some of the benefits associated with breastfeeding [14]. Benefits for the mother include reducing the risks of type 2 diabetes, certain types of breast cancer, and ovarian cancer [14]. 
The literature suggests that breastfeeding is a modifiable factor in reduction of health risks for the mother newborn dyad [15].As such, the knowledge regarding the importance of breastfeeding and strategies to promote breastfeeding are essential.

Although breastfeeding initiation rates are high, breastfeeding continuation rates at six and twelve months remain lower than recommendations, which suggests that mothers want to breastfeed [16] but may be discontinuing early due to inadequate lactation support. Regardless of the reasons a new mother chooses to breastfeed, exclusive breastfeeding requires a strong commitment that can be improved with a strong support system. However, breastfeeding practices may be hindered if a mother is experiencing postpartum fatigue. Although relatively common, postpartum fatigue is poorly researched as to its effect on exclusive breastfeeding rates. This purpose of this study was to determine if there was an effect of postpartum fatigue on exclusive breastfeeding rates at discharge from a mid-sized community hospital in northwestern New Jersey. Additionally the study compared birth methods, postpartum fatigue, and exclusive breastfeeding rates.

\section{Design}

The study design utilized in this study was a quasi-experimental [17]. This design was appropriate as a comparison of postpartum fatigue was made between two groups, mothers who gave birth vaginally versus those who gave birth via cesarean section. Additionally, exclusive breastfeeding rates were compared between vaginal and cesarean births.

\section{Sample and Setting}

The setting was a single community hospital in Northwest New Jersey. A convenience sample of postpartum mothers who had declared their intent to breastfeed exclusively was utilized for this study. This population was selected based on the diverse population and accessibility. The data was collected over a seven-month period, during which time there were 648 live births. Of the 648 live births, 239 mothers met the criteria and 161 surveys were obtained. Of the 161 surveys, 157 surveys were completed.

Inclusion criteria for the study included women over the age of 18, who were English speaking, and who had stated upon admission that they planned to exclusively breastfeed. Women were excluded from the study if they planned a combination feed of both breast and bottle, if the baby was in the NICU, or if they were non-English speaking. Other criteria that excluded women from participating in the study was a history of anxiety or depression including postpartum depression or any type of mental illness. These women were excluded due to the association between postpartum fatigue and postpartum depression noted in the literature.

Whether a mother delivered by cesarean section or vaginal birth, they were offered the opportunity to participate in the study. Typically, postpartum women that deliver by cesarean section stay in the hospital for 3 nights after day of delivery. Women who deliver vaginally spend 2 nights after delivery, barring any complications. The participants were offered to complete the study at no sooner than one day after delivery. All participants were approached in the morning before noon after review of the maternity unit census in order to ensure discharges leaving early were included.

\section{Ethical considerations}

Approval was sought from the Institutional Review Board (IRB) at the participating university and the Research Council at the participating hospital. The nursing staff was informed about the study and advised regarding the inclusion criteria. Information on the study was explained to the patients. An invitation to participate in the study along with the passive consent forms were given to all patients that met criteria. Questions or concerns raised by the study participants were addressed by the researchers. All participants were assured of anonymity through the use of a passive consent. No identifying information was contained on the surveys.

\section{Data Collection Instrument}

The Visual Analogue Scale to Evaluate Fatigue Severity (VAS-F) [18] was administered to participants over a six-month period to measure both fatigue and energy levels. The VAS-F is a selfreport measure consisting of 18 items and two subscales - fatigue and energy. The fatigue subscale ( $\alpha=.94)$ is comprised of 13 items, while the energy subscale ( $\dot{\alpha}=.85)$ is comprised of five items, and higher scores on each scale indicate higher levels of fatigue and energy, respectively. The Cronbach $\alpha$ for this study for the VAS-F was .87. This value indicates that the instrument has a strong internal consistency reliability.

\section{Data Collection Procedures}

After obtaining the daily Maternity Census, the patient charts were examined. The patient history was reviewed for inclusion and exclusion criteria. Once the review was completed, the women meeting study criteria were approached. These women were given an invitation to participate as well as a passive consent. The research study was explained to the mothers and any questions were answered. Once they consented to participate, the surveys were then distributed to any postpartum woman who met inclusion criteria and were over 24 hours postpartum. Two surveys were utilized, a demographic survey that included some basic breastfeeding questions, type of delivery, rooming in, rest period information, and the VAS-F scale.

\section{Data Analysis}

The Statistical Package for Social Sciences (SPSS version 23) was used to analyze the data. Surveys from 161 postpartum women were statistically analyzed to examine the effect of postpartum fatigue on exclusive breastfeeding rates at discharge. Fatigue levels were measured through the VAS-F Scale [18]. This was broken down into three categories: low (<3.39), moderate (3.4-6.79), and high ( $>$ 6.8). An independent samples t-test was used for the data analysis focusing on exclusive breastfeeding, energy, and fatigue.

\section{Results/Findings}

One hundred and fifty-seven of the 161 one mothers completed both surveys in their entirety. Four mothers did not complete all the questions. One hundred forty-five mothers $(89 \%)$ reported they took part in a rest period daily. Of those 145 mothers, only 73 (50.3\%) breastfed exclusively while 71 (49\%) did not breastfeed exclusively and one mother did not respond. Although a higher percentage of mothers exclusively breastfed who took part in the rest period, the results were not statistically significant. This suggests that the rest period did not impact exclusive breastfeeding rates.

In terms of the fatigue scale, $100 \%$ of the participants reported varying degrees of fatigue, with $47 \%$ reporting exclusive breastfeeding during their hospital stay. Low levels of fatigue were reported by 49 mothers $(31.2 \%), 92$ mothers $(58.6 \%)$ reported moderate levels of fatigue, and 16 mothers $(10.2 \%)$ reported high levels of fatigue. No mothers reported having zero or no fatigue and four mothers did not complete their responses. Additionally, of the 49 mothers who reported low levels of fatigue, 21 (13.4\%) reported exclusive breastfeeding while 28 did not exclusively breastfeed. Of the 92 mothers who reported moderate levels of fatigue, 49 (31.2 $\%)$ reported exclusive breastfeeding while 43 did not breastfeed exclusively. In the high fatigue category, $4(2.5 \%)$ mothers of the 16 breastfed exclusively while 12 did not exclusively breastfeed. These findings suggest that postpartum fatigue impacted exclusive breastfeeding rates at discharge.

Differences were also noted between cesarean and vaginal birth. An independent samples T-test was used to compare fatigue levels in vaginal and cesarean births. The results indicated a difference in the scores for vaginal birth $(\mathrm{M}=3.99, \mathrm{SD}=1.74)$ and cesarean birth $(\mathrm{M}=4.71, \mathrm{SD}=1.89)$ which was statistically significant $(\mathrm{p}=0.015)$ (See Table 1).This finding suggests that the mothers who had cesarean births were significantly more fatigued than those that experienced vaginal births. 


\begin{tabular}{|l|l|l|}
\hline \multicolumn{3}{|c|}{ BIRTH METHOD } \\
\hline $\begin{array}{l}\text { Vaginal Birth } \\
\overline{\mathbf{x}} \pm \mathbf{S}\end{array}$ & $\begin{array}{l}\text { Caesarean Section } \\
\overline{\mathbf{x}} \pm \mathbf{S}\end{array}$ & Test and $\mathbf{p}$ value \\
\hline $3.99 \pm 1.74$ & $3.99 \pm 1.74$ & $\mathrm{df}=151, \mathrm{t}=-2.471, \mathrm{p}<0.05$ \\
\hline $4.97 \pm 1.55$ & $4.87+1.55$ & $\mathrm{df}=155, \mathrm{t}=.401, \mathrm{p}>0.05$ \\
\hline
\end{tabular}

Table 1.Comparison of Postpartum Women's Mean VAS-F Scores in Terms of Their Birth Method

Exclusive breastfeeding rates were compared in the 157 women giving birth vaginally and by cesarean section. Findings from a chi squared test showed exclusive breastfeeding rates in women who gave birth vaginally were higher than cesarean section $\left(\chi^{2}=12.47\right.$, $\mathrm{p}<.01)$.Of the 157 women surveyed, 79 gave birth vaginally and
78 delivered by cesarean birth. Of the 79 women who gave birth vaginally, $49(62 \%)$ were exclusively breastfeeding and of the 78 women who gave birth by cesarean section, 30 (38\%) were exclusively breastfeeding (See Figure 1). This suggests that exclusive breastfeeding rates were dependent on birth type.

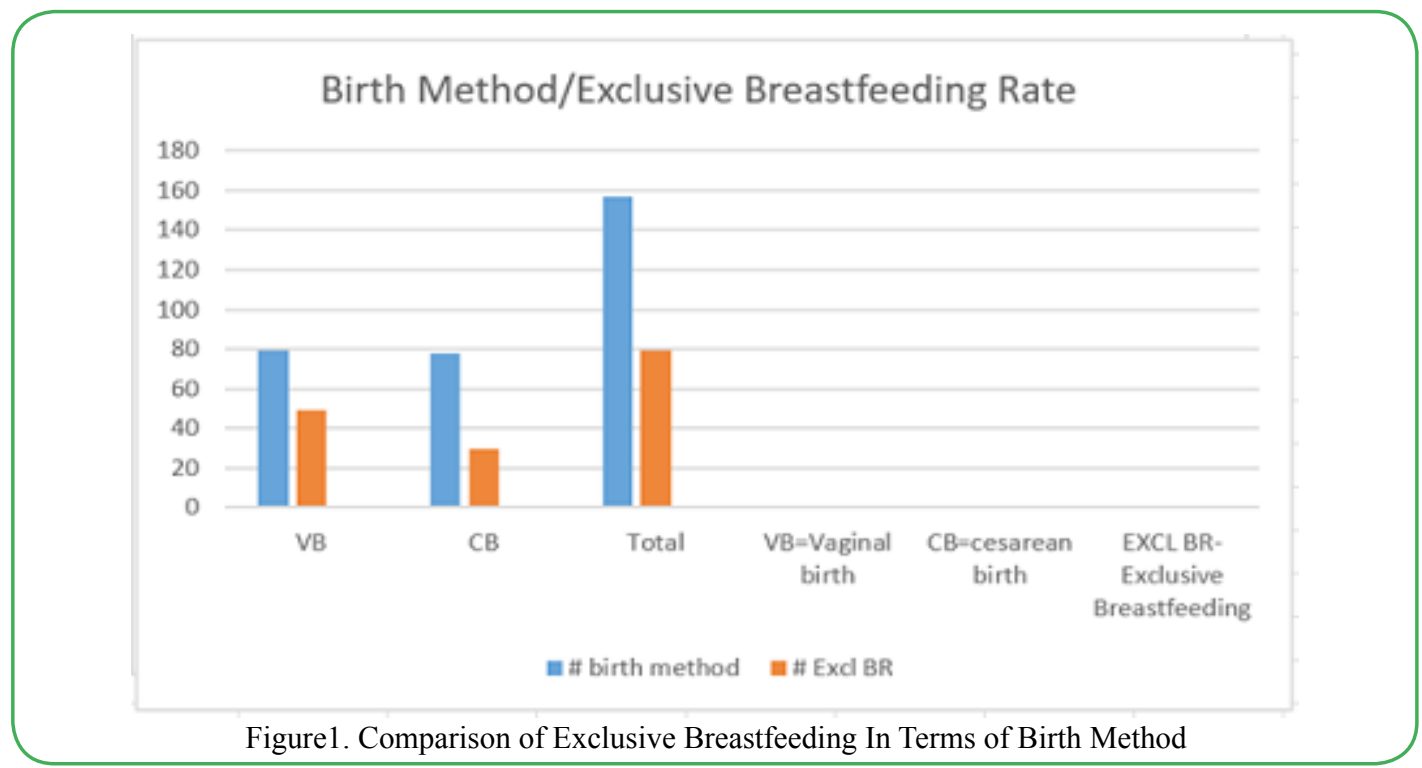

As an incidental finding, mothers who decided to breastfeed prior to pregnancy reported more energy postpartum. An independent samples T-test was used to compare mothers who decided to breastfeed prior to pregnancy $(\mathrm{M}=5.10, \mathrm{SD}=1.48)$ versus those who decided to breastfeed during the pregnancy $(\mathrm{M}=4.54, \mathrm{SD}=1.68)$.
Results indicated a significant difference in their scores $(\mathrm{p}=0.037)$ (See Table 2). These findings suggest that mothers who decided to exclusively breastfeed prior to conceiving had significantly more energy postpartum those who decided to breastfeed during the pregnancy.

\begin{tabular}{|l|l|l|l|}
\hline \multicolumn{4}{|c|}{ DECISION TO BREASTFEED } \\
\hline Sub-Scales & $\begin{array}{c}\text { Prior to Pregnancy } \\
\overline{\mathbf{x}} \pm \mathbf{S}\end{array}$ & $\begin{array}{c}\text { During Pregnancy } \\
\overline{\mathbf{x}} \pm \mathbf{S}\end{array}$ & Test and $\mathbf{p}$ value \\
\hline Fatigue & $4.23 \pm 1.80$ & $4.45 \pm 2.09$ & $\mathrm{df}=154, \mathrm{t}=-.650, \mathrm{p}>0.05$ \\
\hline Energy & $5.10 \pm 1.48$ & $4.54 \pm 1.68$ & $\mathrm{df}=158, \mathrm{t}=2.103 \mathrm{p}<0.05$ \\
\hline
\end{tabular}

\section{Discussion}

\section{Rest Period, Postpartum Fatigue, and Exclusive Breastfeeding}

Another finding involved the impact of the daily rest period on postpartum fatigue. In this study, 145 of 157 mothers took part in a daily rest period of at least an hour with only $50.3 \%$ reporting exclusive breastfeeding. However, no statistical significance was noted. Similarly, in a study conducted at the same hospital in 2014, which examined the effect of a rest period on exclusive breastfeeding $(n=160), 52.5 \%$ of mothers who took part in a daily rest period reported exclusive breastfeeding at discharge [9]. No statistical significance was noted, but a higher percentage of exclusive breastfeeding was noted in the mothers who took part in the rest period [9]. Additionally, statistical significance was noted in relation to mothers perception of breastfeeding difficulty related to exhaustion [9].

The literature also reports that fatigue has been found to negatively impact women during the postpartum period particularly in terms of their own self-care, the care of other family members, and the care of their newborns $[1,2,5]$. A study by Runquist [4] indicated that women referred to postpartum fatigue as an overwhelming and distressing occurrence. The impact of postpartum fatigue, particularly moderate to severe levels could affect infant feeding, particularly breastfeeding. Exclusive breastfeeding rates factoring in levels of fatigue were only $47 \%$ in this study. Exclusive breastfeeding rates in the combined moderate to high categories was only $33.7 \%$ suggesting that postpartum fatigue may have affected exclusive breastfeeding. A study by Lai et al [7] reported that mothers with higher levels of postpartum fatigue perceived greater challenges in regard to infant care and feeding.

\section{Birth Method and Postpartum Fatigue}

Current study findings included statistical significance in the comparison of fatigue levels between vaginal and cesarean birth. Mothers who gave birth via cesarean section were significantly more fatigued postpartum than those who gave birth vaginally. This could 
be related to length of labor prior to cesarean section, incisional pain, blood loss, anemia, and wound healing [5]. These results were also supported by a comparative, descriptive study by Kilic et al. [1] of 383 women utilizing the VAS-F scale who gave birth to healthy newborns in a facility in Turkey. Study findings reported that women giving birth vaginally had lower fatigue levels and higher energy levels than those who gave birth by cesarean section [1].

A descriptive cross-sectional study by Lai et al. [7] reviewed postpartum fatigue, attachment, and baby care activities in 120 women after vaginal and cesarean birth in Taiwan. Postpartum women who were rooming in after cesarean birth reported more fatigue than those after vaginal birth [7]. Physical discomfort from incisional pain could have contributed to this $[7,8,19]$. Conversely, a cross sectional study by Hsieh et al. [8] of 326 postpartum women in Taiwan who gave birth vaginally reported that women with more perineal trauma and longer duration of second stage of labor experienced higher levels of fatigue postpartum [8]. This could be due to perineal pain impacting adequate rest postpartum [8].

\section{Birth Method and Exclusive Breastfeeding}

In the current study, mothers who gave birth by cesarean section were less likely to exclusively breastfeed as compared to those who gave birth vaginally. This was supported by several previous studies. A study of over 3000 women by [10] using data from Infant Feeding Practices (2005-2006) noted no significance between birth method and breastfeeding initiation. However breastfeeding continuation was found to be less likely in women who gave birth via cesarean birth, particularly so if an emergency cesarean birth or if labor was induced [10].

Breastfeeding behaviors according to birth method were also studied postpartum by Cetisli, Arkan, \& Top [20]. Study participants included 175 mothers, 83 who gave birth vaginally and 92 who had a cesarean delivery in Turkey. Mothers who gave birth by cesarean birth reported more difficulties with breastfeeding than those mothers who delivered vaginally [20,21]. In addition to the commonly seen discomforts after cesarean birth, difficulty with positioning for breastfeeding was also noted $[20,21]$. As well, a study regarding postoperative pain after cesarean birth and breastfeeding noted breastfeeding was impacted negatively by high levels of pain [19] .

\section{Decision to Breastfeed}

Another finding from this study was that women who had chosen to breastfeed prior to the pregnancy reported higher levels of energy postpartum than those who decided during the pregnancy. Many factors are involved in the decision-making process: age, culture, education, social support, self-confidence in ability, and previous breastfeeding history among others [22]. Since reduced energy levels could relate to fatigue which could impact breastfeeding success, this suggests that higher energy levels postpartum could promote increased initiation and continuation of breastfeeding. The significance of the decision to breastfeed prior to pregnancy also suggests early prenatal education may raise awareness for those women contemplating a future pregnancy and may sway their decision to breastfeed [22] Although breastfeeding education and social support are important factors in promoting breastfeeding, a multifaceted approach has been found to be more successful [22].

Less information is available about making the decision to breastfeed prior to pregnancy and breastfeeding success. In this study of the 157 participants, 112 women $(71.3 \%)$ made the decision to breastfeed prior to pregnancy. Forty-five women (28.7\%) made the decision during the pregnancy. Of the 112 women who decided prior to pregnancy, $56(50 \%)$ reported exclusive breastfeeding. Of those 45 women who decided during the pregnancy, $22(49 \%)$ breastfed exclusively. This suggests that the decision to breastfeed prior to pregnancy did not have an overall impact on exclusive breastfeeding rates at discharge. However, this is an avenue to be further evaluated with a larger sample size and a comparison between primiparas and multiparas.

\section{Limitations}

There are several limitations to this study. First, the study was conducted in a single community hospital in northwestern New Jersey $(n=157)$ so the results may not be generalizable. The hospital was on a high flu alert so all visitors were restricted. No children were allowed to visit which could have impacted the amount of rest periods mothers experienced which could have affected the perception of fatigue. Since 145 mothers took part in a daily rest period, this could have reduced postpartum fatigue levels. At 24 hours postpartum mothers who gave birth by cesarean section could have experienced more pain, which could have affected perception of fatigue and impacted breastfeeding success.

Additionally, a potential existed for bias because the researcher was a lactation consultant. Data collection took place in the hospital where the individuals conducting the study work which could influence the staff's interaction with the breastfeeding mothers. Staff members were advised that the interviewers were not functioning in the role of a lactation consultant or staff nurse but as researchers'. No data was collected during working hours. The research agenda, assumptions, and participant selection criteria were stated up front. Lastly, the sample size was small and was primarily Caucasian ( $\mathrm{n}=$ 103) which limits the consideration of other races. A larger sample could have elicited other results from different perspectives.

\section{Conclusions and Implications for Future Research}

Postpartum fatigue, in varying levels (low, moderate, and high), was reported in $100 \%$ of the study participants. This is higher than the $64 \%$ noted in the meta-analysis conducted by Badr and Zauszniewski from 1996 to 2016 [2]. Similarly, a study of active duty military women by noted $62 \%$ of women experienced moderate fatigue and $18.3 \%$ experienced high fatigue levels on the second postpartum day [23]. Although much has been written in the literature about postpartum fatigue, the study findings may suggest that postpartum fatigue may be underreported.

Postpartum fatigue is a ubiquitous condition that impacts all women during the postpartum period to some degree. For more than two decades, postpartum fatigue has been ranked in the top five concerns impacting women during the postpartum period [5] and has been associated with postpartum depression $[5,24]$. Prenatal education should include information about postpartum fatigue, strategies of coping with postpartum fatigue, and the importance of rest periods [3]. During the postpartum hospitalization, assessment for postpartum fatigue should be addressed as well as suggested coping strategies which could include flexible rooming in policies $[7,22$, 25], and designated rest periods [9].

Although there was no statistical significance between postpartum fatigue and exclusive breastfeeding rates in this study, only $47 \%$ of mothers were breastfeeding exclusively at discharge. Breastfeeding is well known to be the optimal way to provide nutrition to infants through the first year $[12,16]$. As such, breastfeeding remains the healthiest choice for mother and baby and promoting ways to improve exclusivity needs to remain a high societal priority. Barriers that impede the success of exclusive breastfeeding need to be identified in order to provide the skills necessary to promote a strong foundation of health for babies as well as improved health outcomes for the mothers that are in alignment with the Healthy People 2020 goals. Hospital policies should be reviewed and revised to include rest periods for mothers, education on postpartum fatigue, strategies for coping with postpartum fatigue, anticipatory guidance, and lactation support that reduce barriers to breastfeeding, thus promoting exclusive breastfeeding.

Future recommendations for studies include strategies for coping with postpartum fatigue along with strategies that improve exclusive breastfeeding rates. As well, additional studies with a larger sample in multiple hospitals are necessary to explore the role of postpartum 
fatigue on early discontinuation of breastfeeding, particularly exclusive breastfeeding. This study focused on the influence of postpartum fatigue on exclusive breastfeeding rates at discharge, future research considerations should include the influence of postpartum fatigue on exclusive breastfeeding during the postpartum period before and after discharge. Further studies regarding birth type and postpartum fatigue should include comparisons between primiparas and multiparas.

\section{Acknowledgements}

The primary researcher received Assigned Release Time from William Paterson University to conduct the study. The researchers would like to acknowledge the postpartum women who gave their time to participate in this study.

Conflicts of Interest: The authors declare no conflicts of interest.

\section{References}

1. Kilic, M., Ozorhan, E., Apay, S., Capik, A., Agapinar, S., \& Özkan, H. (2015). Comparison of fatigue levels of postpartum women according to the birth method. International Journal of Caring Sciences 8(1):124-130.

2. Badr, H., \& Zauszniewski, J. (2017). Meta-analysis of the predictive factors of postpartum fatigue. Applied Nursing Research 36:122-127.

3. McQueen, A., \& Mander, R. (2003). Tiredness and fatigue in the postnatal period. Issues and Innovations in Nursing Practice, 42 (5): 463-469.

4. Runquist, J, (2007). Persevering through postpartum fatigue. Journal of Obstetrics, Gynecologic, and Neonatal Nursing, 36(1):28-37.

5. Troy, N. (2003). Is the significance of postpartum fatigue being overlooked in the lives of women? The American Journal of Maternal Child Nursing, 28(4):262-257.

6. Troy, N., \& Dalgas-Pelish, P. (2003). The effectiveness of a selfcare intervention for the management of postpartum fatigue. Applied Nursing Research, 16 (1): 38-45.

7. Lai, Y., Hung, C., Stocker, I, Chan, T., \& Liu, Y. (2014). Postpartum fatigue, baby care activities, and maternal attachment of vaginal and cesarean births following rooming in. Applied Nursing Research, 28(2):116-120.

8. Hsieh, C., Chen, C., Han, T., Lin, P., Chiu, H. (2018). Factors influencing postpartum fatigue in vaginal-birth women: Testing a Path Model. The Journal of Nursing Research, 26(5):332-339.

9. Phillips, K., Malig, K., \& DePue, L. (2015). The influence of quiet time on exclusive breastfeeding rates at discharge. International Journal of Nursing Didactics, 5 (7): 1-6.

10. Ahluwalia, I., Li, R., \& Morrow, B. (2012). Breastfeeding practices: Does method of delivery matter? Maternal Child Health Journal, 16:S231-S237.

11. Kurth, E., Kennedy, H., Spichiger, E., Hösli, I., \& Stutz, E. (2011). Crying babies, tired mothers: What do we know? A systematic review. Midwifery, 27:187-194. doi.10.1016/j. midw.2009.05.012.

12. American Academy of Pediatrics [AAP]. (2012). Breastfeeding and the use of human milk

13. Centers for Disease Control [CDC]. (2019). About breastfeeding.

14. Office on Women's Health. (2019). Making the decision to breastfeed.

15. Stuebe A. (2009). The risks of not breastfeeding for mothers and infants. Reviews in Obstetrics \& Gynecology, 2(4):222-231.

16. Centers for Disease Control [CDC]. (2018). Breastfeeding report card.
17. Polit, D., \& Beck, C. (2017). Essentials of nursing research: Appraising evidence for nursing practice (9th Ed). Phila, PA: Wolters Kluwer

18. Lee, K., Hicks, G., \& Nino-Murcia, G. (1990). Reliability and validity of a scale to assess fatigue. Psychiatry Research, 36: 291-298.

19. Karlstrom, A., Engstrom-Olofsson, R., Norbergh, K., Gjoling, M., \& Hildingsson, I. (2007). Postoperative pain after cesarean birth affects breastfeeding and infant care. Journal of Obstetrics, Gynecologic, and Neonatal Nursing, 36(5):430-440. doi:10.1111/J.1552-6909.2007.00160.x

20. Cetisli, N., Arkan, G., \& Top, E. (2018). Maternal attachment and breastfeeding behaviors according to type of delivery in the immediate postpartum period. Rev Assoc Med Bras, 64(2): 164169.

21. Zanardo, V., Svegliado, G., Cavallin, F., Giustardi, A., Cosmi, E., \& Litta, P. et al. (2010). Birth, 37 (4): 275-279.

22. Radzyminski, S., Callister, L. (2016). Mother's beliefs, attitudes, and decision making related to infant feeding choices. The Journal of Perinatal Nursing, 25 (1): 18-28.

23. Rychnovsky, J. (2007). Postpartum fatigue in the activeduty military woman. Journal of Obstetric, Gynecologic, and Neonatal Nursing, 36(1): 38-46.

24. Thomas, K., Spieker, S. (2016). Sleep, depression, and fatigue in late postpartum. The American Journal of Maternal Child Nursing, 41(1):104-109.

25. Tsuchiya, M., More, E., Iwata, H., Sakajo, A., Maehara, K, Ozawa, H....Sacki, A. (2015). Fragmented sleep and fatigue during postpartum hospitalization in older primiparous women. Nursing and Health Sciences, 17:71-76. Doi:10.1111/nhs. 12157 